\title{
The Reaction of Heterocyclic Nucleophiles and the DABCO Salts of the Baylis-Hillman Acetates
}

\author{
Ji Hyeon Gong, Hỵoung Rae Kim, ${ }^{\dagger}$ Eung K. Ryu, ${ }^{\dagger}$ and Jae Nyoung Kim ${ }^{\star}$ \\ Department of Chemistry and Institute of Basic Science, Chonnam National University, Kuangiu 500-757, Korea \\ ${ }^{\dagger}$ Bioorganic Science Division, Korea Research Institute of Chemical Technology: \\ P.O. Box 107, Yusong, Daejon 305-600, Korea \\ Received Febriany 23, 2002
}

Keywords : Baylis-Hillman acetates. DABCO. Allylic substitution, Rearrangement, Heterocyclic nucleophiles.

Recently, we and others have reported on the reaction of the DABCO (1.4-diazabicyclo[2.2.2]octane) salts of the Baylis-Hillman acetates with some nucleophiles. ${ }^{1,2}$ In the reaction we could obtain the allylic substitution products (nucleophile at the secondary position of prodtct) in good yields. Examined nucleophiles included sodium borohydride, ${ }^{\text {la }}$ potassium cyanide ${ }^{\mathrm{lb}}$ and $p$-toluenesulfonamide. ${ }^{\mathrm{le}}$

As a continuous work we examined the reaction of the $\mathrm{DABCO}$ salts of the Baylis-Hillman acetate and some heterocycles such as uracil or thymine. However, we could obtain the rearrangement product (nucleophile at the primary: position of prodtct $)^{2 c}$ as the major. Drewes et al. have reported the reaction of Baylis-Hillman acetate and 2formylimidazole. ${ }^{\text {?c }}$ This is the only report that dealt with the heterocyclic nucleophile, where either allylic substitution product or rearrangement product can be selectively obtained by controlling the reaction conditions. Thus, we intended to study on the reaction of the DABCO salts of BaylisHillman acetates with various heterocyclic nucleophiles in order to obtain some insights on the reaction pathway depending on the nucleophiles and reaction conditions.

As shown in Scheme 1, the reaction proceeded via the DABCO salt of the Baylis-Hillman acetate. The products were either allylic substitution product 3 or the rearrangement one $\mathbf{4}$ depending on the nucleophiles. We used BaylisHillman acetate $\mathbf{1}$ as a model substrate and isatin (2a), benzotriazole ( $2 \mathrm{~b}$ ), phthalimide (2c), oxindole (2d), barbituric acid (2e), uracil (2f), thymine ( $2 \mathrm{~g}$ ), 1-hydroxybenzotriazole (2h) and 1,2,4-triazole (2i) as heterocyclic nucleophiles. The results are summarized in Table 1 and 2 . We could obtain the

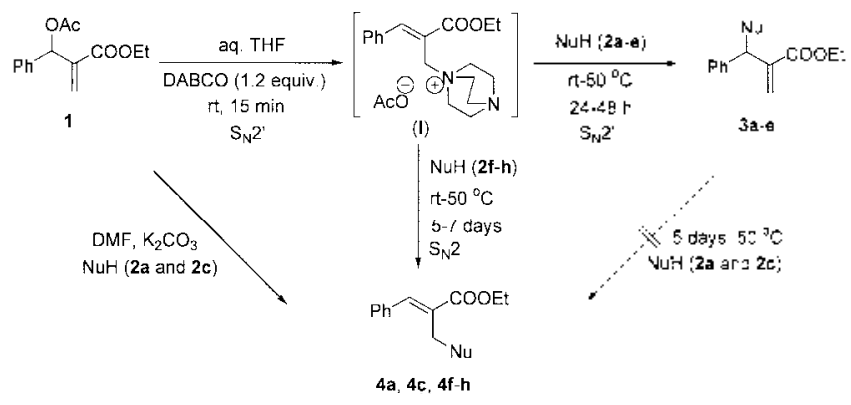

Scheme 1 allylic substitution products 3a-e selectively in $50-91 \%$ yields for $\mathbf{2 a - e}$ within relatively short time (Table 1). While, rearrangement products $\mathbf{4 f - h}$ were isolated as the major (51$63 \%$ ) in the cases of $\mathbf{2 f - h}$ (Table 2). For the synthesis of $\mathbf{4 f - h}$ somewhat long reaction time was needed.

We could tentatively propose the regiochemistry as follows (Scheme 1). When the nucleophiles have good reactivity toward the DABCO salts (I) under the reaction conditions, $\mathrm{S}_{\mathrm{N}} 2^{\prime}$ type reaction proceeded to give $3 .{ }^{1}$ Whereas when we used nucleophiles such as $\mathbf{2 f}-\mathbf{h}$, rearranged products $\mathbf{4}$ might be formed via either the corresponding 3 by successive intermolecular $\mathrm{S}_{*} 2^{1}$ reaction by the same nucleophile or direct $S_{*} \leqslant 2$ type reaction from (I). Thus, we examined the

Table 1. Synthesis of allylic substitution products 3

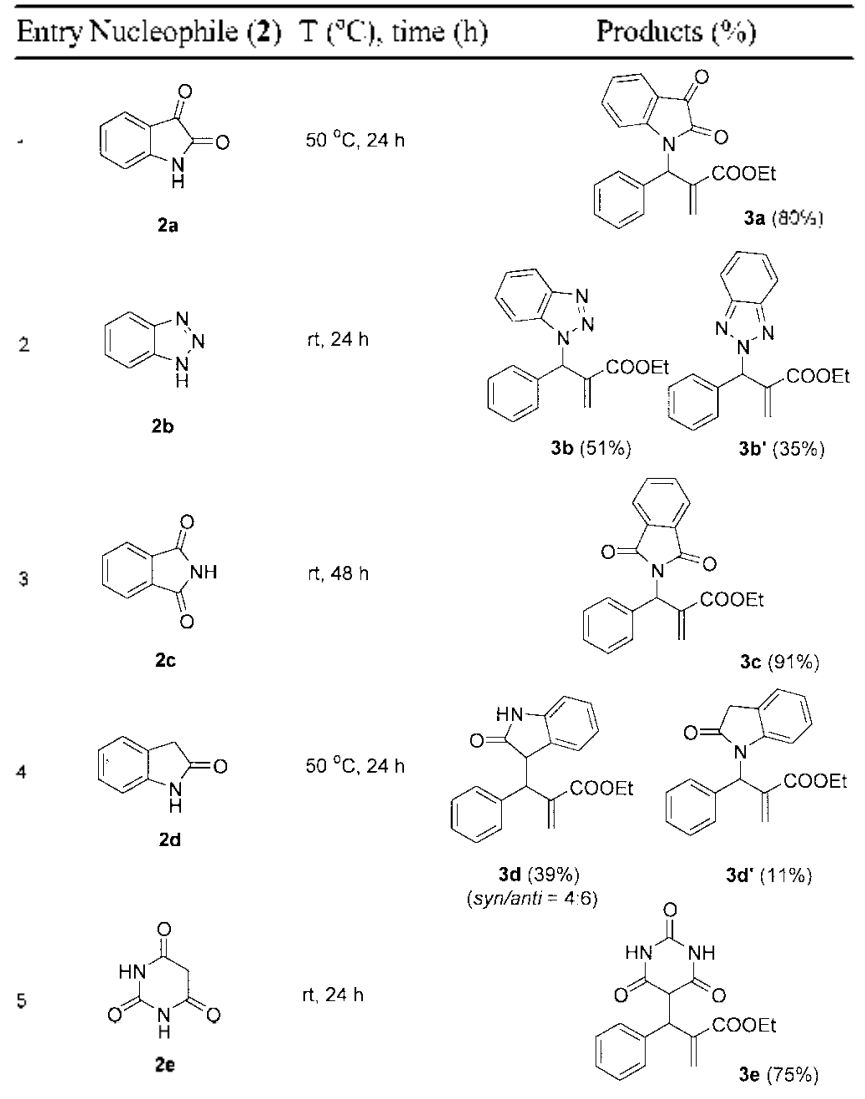


Table 2. Synthesis of rearrangement products 4

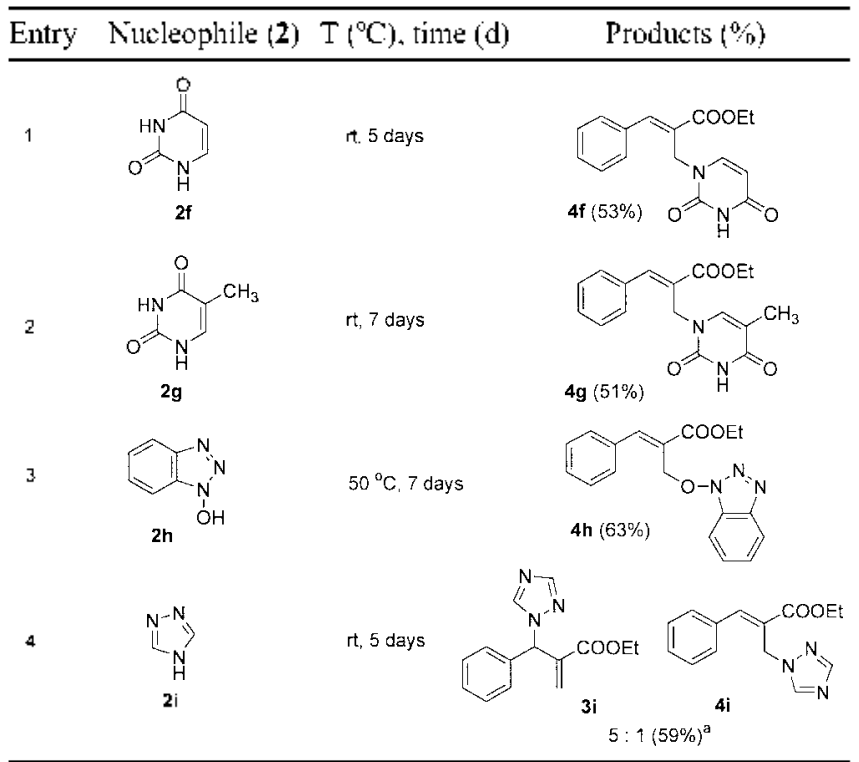

The ratio was determined from ${ }^{1} \mathrm{H}$ NMR spectrum and the position of nitrogen atom is arbitrary.

reaction of $\mathbf{3 a}$ and $\mathbf{3 c}$ in the presence of isatin (2a) or phthalimide $(2 \mathfrak{c})$ under the same reaction conditions. However, the formation of $4 a$ and $4 \mathbf{c}$ was not detected ( 5 days at $50^{\circ} \mathrm{C}$ in aqueous THF in the presence of $\mathrm{DABCO}$ ). From the results, we tentatively conclude that 4 might be formed from (I) by a $S_{k} 2$ reaction directly. As shown in Scheme 1, rearrangement products $\mathbf{4 a}(90 \%)$ and $\mathbf{4 c}(84 \%)$ could be prepared simply by changing the reaction conditions (the use of $\mathrm{K}_{2} \mathrm{CO}_{3}$ in DMF).

In the case of benzotriazole (entry 2 in Table 1), competition between $N_{1}$-attack and $N_{2}$-attack was observed. The structures of $\mathbf{3} \mathbf{b}$ and $\mathbf{3} \mathbf{b}^{\prime}$ could be easily discernable by the ${ }^{13} \mathrm{C}$ NMR spectra. Due to the symmetry of benzotriazole moiety in $\mathbf{3} \mathbf{b}^{\prime}$ only 13 peaks of carbon were observed. ${ }^{3}$ When we used oxindole (entry 4 in Table 1), competition between $C$-attack and $N$-attack was observed. The structures of $3 \mathbf{d}$ and $3 \mathbf{d}$ ' were also deduced from their ${ }^{l} \mathrm{H}$ NMR spectra easily. The compound $\mathbf{3 d}$ exists as a syn/anti mixture (4:6 ratio). ${ }^{3}$ In the case of 1,2,4-triazole (entry 4 in Table 2) $\mathbf{3 i}$ and $\mathbf{4 i}$ was generated as a $5: 1$ ratio. We could not separate them in pure state. The precise structure (the position of nitrogen atom) of $\mathbf{3 i}$ and $\mathbf{4 i}$ was not proved until now.

As a conclusion, we examined the reaction of the $\mathrm{DABCO}$ salt of the Baylis-Hillman acetate and various heterocyclic nucleophiles. Depending on the nature of nucleophiles and reaction conditions, regiochemistry of the nucleophilic substitution reaction could be controlled. Further studies on the relationships between the regiochemistry of products and nucleophiles are currently underway.
Acknowledgment. This work was supported by the grant (No. R02-2000-00074) from the Basic Research Program of the Korea Science \& Engineering Foundation.

\section{References and Notes}

1. (a) Im. Y. J.; Kim. J. M.; Mun. J. H.; Kim. J. N. Bull. Korean Chem. Soc. 2001, 22. 349, (b) Chung. Y. M.; Gong. J. H.; Kim. T. H.; Kim, J. N. Tetrahedron Lett. 2001, 42. 9023. (c) Kim, J. N,; Lee, H. J.: Lee, K. Y.: Gong, J. H. Synlett 2002, 173.

2. (a) Bastvaiah, D.: Kumaragurubaran, N. Tetrahedron Lett. 2001, 42, 477. (b) Basavaiah, D.; Kumaragurubaran, N.; Sharada, D. S. Tetwahedron Lett. 2001. 42. 85. (c) Drewes, S. E.; Horn, M. M.; Ramesar, N, Symth. Conmun. 2000, 30. 1045 .

3. Some selected spectroscopic data of products are as follows. 3a: clear oil; $\mathrm{IR}(\mathrm{KBr}) 1737,1684 \mathrm{~cm}^{-1}$ : 'H NMR $\left(\mathrm{CDCl}_{3}\right) \delta 1.10$ $(\mathrm{t}, J=7.2 \mathrm{~Hz}, 3 \mathrm{H}), 4.10(\mathrm{~m}, 2 \mathrm{H}), 5.57(\mathrm{~d}, J=1.8 \mathrm{~Hz}, 1 \mathrm{H}), 6.37(\mathrm{~s}$, $1 \mathrm{H}), 6.52(\mathrm{~d}, J=1.8 \mathrm{~Hz}, 1 \mathrm{H}), 6.75(\mathrm{~d}, J=8.1 \mathrm{~Hz}, 1 \mathrm{H}), 6.99(\mathrm{t}, J=$ $7.8 \mathrm{~Hz} .1 \mathrm{H}), 7.24-7.38(\mathrm{~m}, 6 \mathrm{H}) .7 .54(\mathrm{~d} . J=7.5 \mathrm{~Hz}, 1 \mathrm{H}) ;{ }^{13} \mathrm{C}$ NMR $\left(\mathrm{CDCl}_{3}\right) \delta 12.96,55.59$. 60.34. 111.52. 116.90, 122.67, 124.35, 126.93. 127.44. 127.96, 128.53. 134.59, 136.70. 137.16, $149.94,157.06,164.37,181.82$

3b: clear oil; $\mathrm{IR}(\mathrm{KBr}) 1719 \mathrm{~cm}^{-1}$; ${ }^{\mathrm{H}} \mathrm{NMR}\left(\mathrm{CDCl}_{3}\right) \delta 1.03\left(\mathrm{t}_{1} J=\right.$ $7.1 \mathrm{~Hz}, 3 \mathrm{H}), 4.04(\mathrm{~m}, 2 \mathrm{H}), 5.4 \mathrm{l}(\mathrm{s}, 1 \mathrm{H}), 6.55(\mathrm{~s}, 1 \mathrm{H}), 6.98(\mathrm{~s}, 1 \mathrm{H})$, $7.18-7.31(\mathrm{~m}, 8 \mathrm{H}) .7 .97(\mathrm{~d}, J=8.0 \mathrm{~Hz}, 1 \mathrm{H}) ;{ }^{3} \mathrm{C}$ NMR $(\mathrm{CDCl})$ $13.81,61.25,62.23 .109 .84,119.94,123.91 .127 .37,127.91$. $128.63,128.85,129.75,132.91 .135 .91 .138 .61 .145 .99,165.14$ $3 b^{\prime}$ : clear oil: $\mathrm{IR}(\mathrm{KBr}) 1720 \mathrm{~cm}^{-1}$; 'H NMR (CDCl $) \delta 1.02$ (t, $J=$

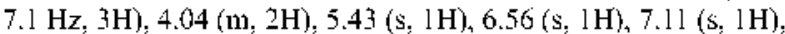
$7.20-7.29(\mathrm{~m}, 7 \mathrm{H}), 7.77-7.81(\mathrm{~m}, 2 \mathrm{H}){ }^{13} \mathrm{C} \mathrm{NMR}\left(\mathrm{CDCl}_{3}\right) \delta 12.89$ $60.22,68.72$. 117.28, 125.43. 126.98. 127.72, 127.81, 128.92. $135,03,137.78,143,23,164,09$.

3d: clear oil; IR (KBr) $1749.1696 \mathrm{~cm}^{-1} ; 4: 6 \mathrm{syn} /$ anti mixture; ${ }^{1} \mathrm{H}$ NMR (CDCl $) \delta 1.14$ (t. $J=7.2 \mathrm{~Hz} .1 .2 \mathrm{H}$ ). 1.26 (t. $J=7.2 \mathrm{~Hz}$, $1.8 \mathrm{H}), 4.01-4.29(\mathrm{~m}, 3 \mathrm{H}), 4.57(\mathrm{~d}, J=6.6 \mathrm{~Hz}, 0.6 \mathrm{H}), 4.83(\mathrm{~d}, J=$ $3.9 \mathrm{~Hz}, 0.4 \mathrm{H}), 5.59(\mathrm{~s}, 0.6 \mathrm{H}), 5.64(\mathrm{~s}, 0.4 \mathrm{H}), 6.41(\mathrm{~s}, 0.4 \mathrm{H}), 6.44$ $(\mathrm{s}, 0.6 \mathrm{H}), 6.77-7.26(\mathrm{~m}, 9 \mathrm{H}), 8.80(\mathrm{~s}, 0.6 \mathrm{H}), 8.90(\mathrm{~s}, 0.4 \mathrm{H}),{ }^{13} \mathrm{C}$ NMR (CDCl $) \delta 13.91 .14 .08 .46 .76 .48 .19,48.31,49.95,60.85$. $60.97,109.72,109.79,121.78$. 122.01. 124.86. 125.26. 126.96. 127.11. 127.15. 127.81, 128.01. 128.04. 128.23, 128.28, 128.53 . $129.02,138.54,138.86,140.03,141.48,141.72,166.57,166.74$, 178.46 .

3d': clear oil; IR (KBr) $1776,1721,1656 \mathrm{~cm}^{-1}$ : ${ }^{1} \mathrm{H}$ NMR (CDCl $\delta 1.15$ (t. $J=7.2 \mathrm{~Hz}, 3 \mathrm{H}) .3 .59$ (s. $2 \mathrm{H}), 4.15$ (q. $J=7.2 \mathrm{~Hz} .2 \mathrm{H}$ ), 5.59 (d. $J=1.2 \mathrm{~Hz}, 1$ H). 6.53 (d. $J=1.2 \mathrm{~Hz}$. $1 \mathrm{H}$ ). 6.63 (t. $J=1.2$ $\mathrm{Hz}, 1 \mathrm{H}) .6 .70-7.33(\mathrm{~m} .9 \mathrm{H}) ;{ }^{13} \mathrm{C}$ NMR $\left.(\mathrm{CDCl})_{3}\right) \delta 13.93 .35 .51$. $55.61,61.09,110.98,122.11,124.35,124.40,127.49,127.88$, $128.10,128.47,128.63,136.71,138.59,144.03,165.71,174.90$. 4a: clear oil; IR (KBr) $1747,1738 \mathrm{~cm}^{-1}$ : 'H NMR $\left(\mathrm{CDCl}_{3}\right) \delta 1.15$ (t. $J=7.2 \mathrm{~Hz}, 3 \mathrm{H}), 4.12$ (q. $J=7.2 \mathrm{~Hz} .2 \mathrm{H}), 4.75$ (s. $2 \mathrm{H}) .6 .48$ (d. $J=7.8 \mathrm{~Hz}, 1 \mathrm{H}), 6.93(\mathrm{t}, J=7.2 \mathrm{~Hz} .1 \mathrm{H}) .7 .20-7.39(\mathrm{~m}, 7 \mathrm{H}), 7.91$ (s, $1 \mathrm{H}) ;{ }^{13} \mathrm{C}$ NMR $\left(\mathrm{CDCl}_{3}\right) \delta 14.14 .37 .28,61,48.117 .07 .117 .46$. $123.51,125.06,125.93,128.72,128.85,129.26,134.04,138.15$, $144.13,150.56,157.84,166.28,183.05$.

4f: white solid, mp 114-116 ${ }^{\circ} \mathrm{C}$ : IR (KBr) 3153, 3074, 2873, 1721, $1658 \mathrm{~cm}^{-1},{ }^{1} \mathrm{H}$ NMR $\left(\mathrm{CDCl}_{3}\right) \delta 1.26(\mathrm{t}, J=7.1 \mathrm{~Hz} .3 \mathrm{H}), 4.21$ (q. $J$ $=7.1 \mathrm{~Hz}, 2 \mathrm{H}) .4 .74$ (s. $2 \mathrm{H}) .5 .55(\mathrm{~d}, J=8.0 \mathrm{~Hz}, 1 \mathrm{H}) .7 .15(\mathrm{~d} . J=$ $8.0 \mathrm{~Hz}, 1 \mathrm{H}), 7.31-7.38(\mathrm{~m} .5 \mathrm{H}) .7 .98(\mathrm{~s}, 1 \mathrm{H}), 9.11(\mathrm{~s}, \mathrm{NH}, 1 \mathrm{H}) ;{ }^{13} \mathrm{C}$ NMR $\left(\mathrm{CDCl}_{3}\right) \delta 12.39,42.89,59.82,99.83,123.95,127.06$, $127.31,127.82,132.10,142.39,143.93,148.97,161.87,164.71$. 